\title{
EDITORIAL
}

\section{The autoimmune tautology}

\author{
Juan-Manuel Anaya* \\ See related research by Eyre et al., http://arthritis-research.com/content/12/5/R175
}

\begin{abstract}
Although autoimmune diseases exhibit contrasting epidemiological features, pathology, and clinical manifestations, three lines of evidence demonstrate that these diseases share similar immunogenetic mechanisms (that is, autoimmune tautology). First, clinical evidence highlights the co-occurrence of distinct autoimmune diseases within an individual (that is, polyautoimmunity) and within members of a nuclear family (that is, familial autoimmunity). Second, physiopathologic evidence indicates that the pathologic mechanisms may be similar among autoimmune diseases. Lastly, genetic evidence shows that autoimmune phenotypes might represent pleiotropic outcomes of the interaction of non-specific disease genes.
\end{abstract}

Autoimmune diseases are chronic conditions initiated by the loss of immunological tolerance to self-antigens and represent a heterogeneous group of disorders that afflict specific target organs or multiple organ systems. The chronic nature of these diseases places a significant burden on the utilization of medical care, direct and indirect economic costs, and quality of life. Almost all autoimmune diseases disproportionately affect middleaged women and are among the leading causes of death for this group of patients. With increasing age of patients, the female-to-male ratio for autoimmune diseases becomes more prominent.

The autoimmune tautology refers to the fact that autoimmune diseases share several clinical signs and symptoms, physiopathologic mechanisms, and genetic factors and this fact indicates that they have a common origin (Table 1). In the previous issue of Arthritis Research \& Therapy, Eyre and colleagues [1] report that

*Correspondence: anayajm@gmail.com

Center for Autoimmune Diseases Research (CREA), School of Medicine and Health Sciences, Universidad del Rosario, Carrera 24 \# 63C-69, 111221, Bogota, Colombia variation within the TAGAP gene, at $6 \mathrm{q} 25.3$, is associated with three autoimmune diseases, namely rheumatoid arthritis, type 1 diabetes, and coeliac disease, in Caucasians. In addition, the authors confirmed other loci associated with these diseases in their population: CTLA-4, the IL2-21 region, 6q23 (TNFAIP3), SH2B3, $P R K C Q$, and MMEL1 [1].

The impact of genetic predisposition on susceptibility to autoimmune diseases was first identified by the analysis of disease concordance rates in monozygotic twins (concordance rates ranged from about 15\% to 57\%) [2]. The decrease in the concordance rates of siblings compared with the rate among monozygotic twins supports the presence of multiple genes contributing to the autoimmune phenotype onset.

A primary characteristic of complex diseases is that affected individuals tend to cluster in families (that is, familial aggregation, also referred to as recurrence risk). Recurrent associations of autoimmune diseases in family members of patients have been reported. Although nongenetic factors may have an effect on familial aggregation, shared genetic factors, in fact, may be the most likely cause for this aggregation [3]. However, segregation of autoimmunity is fairly well understood.

Becker and colleagues [4], on the basis of previous autoimmune disease linkage studies, reported 18 common non-major histocompatibility complex loci clusters and hypothesized a shared genetic basis for the autoimmune trait. Since then, several reports, including that of Eyre and colleagues, have confirmed that autoimmune phenotypes might represent pleiotropic outcomes of nonspecific disease genes [1,5-8]. However, the finding of a polymorphism associated with a trait is not complete until the functional relevance is examined and its biological effect on such a trait is understood.

It is noteworthy that not all autoimmune diseases share the same genetic susceptibility. Thus, the genetic risk factors for autoimmune diseases might well consist of two forms: those common to many autoimmune diseases and those specific to a given disorder. Combinations of common and disease-specific alleles at HLA and nonHLA genes, in interaction with epigenetic and environmental factors over time (that is, gluten, tobacco, 


\section{Table 1. Shared characteristics provide evidence that autoimmune diseases have a common origin}

Preponderance of females.

Similar pathophysiology.

Systemic autoimmune diseases have similar signs and symptoms.

Severity is inversely related to the age of onset.

Similar environmental agents (that is, tobacco, Epstein-Barr virus, and so on) may influence autoimmune diseases

Ancestry might influence the clinical presentation.

Common genetic factors (for example, HLA-DRB1, STAT4, IRF5, PTPN22, CD226, FCGR3B, CD44, and TAGAP).

Polyautoimunity (that is, autoimmune diseases may co-occur within patients).

Aggregation. Familial autoimmunity (that is, diverse autoimmune diseases on multiple members of a nuclear family) seems to be more frequent than familial autoimmune disease (that is, one specific autoimmune disease in various members of a nuclear family).

The same pharmacological agent (biologic and non-biologic) may be useful in treating diverse autoimmune diseases.

Epstein-Barr virus, cytomegalovirus, and so on), will determine the final clinical autoimmune phenotype [9]. Yet only around $10 \%$ to $15 \%$ of the inherited risk for autoimmune diseases can be explained at present $[7,8]$. Most of the common variants individually or in combination confer relatively small increments in risk (1.1- to 1.5 -fold) and explain only a small proportion of heritability (that is, the proportion of phenotypic variation in a population that is attributable to genetic variation among individuals). The amount of heritability depends on the investigated population because variations in both additive and non-additive genetic factors and the environmental variance are specific to the population [10]. As a corollary, genetic results should be confirmed in different populations [11]. There are several examples in which polymorphisms influencing the risk for developing autoimmune diseases in a particular population are not replicated in another one. Besides the inner characteristic of the studied population and gradients of allelic frequencies among populations, other explanations for the missing heritability have been suggested: much larger numbers of variants of smaller effect yet to be found; rarer variants (possibly with larger effects) that are poorly detected by available genotyping arrays that focus on variants present in $5 \%$ or more of the population; structural variants poorly captured by existing arrays; low power to detect gene-gene interactions; and failure to account adequately for a shared environment among relatives [12]. Potential sources of missing heritability and research strategies to elucidate the genetics of complex diseases have been proposed [12]. It is evident, however, that the rapid development of DNA sequencing technologies and the continuously improving depth of coverage of polymorphisms by DNA chips for genome-wide association studies will contribute to an extremely expansive phase of genetic research.

Identification of genetic causes of autoimmune diseases will enhance our understanding of the mechanisms common to multiple autoimmune diseases and to specific ones and will assist in providing increasing predictive capacity to identify subjects at risk for these devastating diseases and to identify new therapeutic interventions. Thus, in the near future, we should be able to predict which families or individuals within families may have a higher predisposition to develop an autoimmune phenotype by inspecting his or her family medical history, ancestry, and genetic and autoantibody profile [3]. Although much progress has been made in identifying genetic factors that influence susceptibility to autoimmune diseases, progress is lacking in identifying those factors that influence disease progression or complications. It is conceivable that only a subset of the genetic factors that influence susceptibility to disease also influence disease progression and complications.

Competing interests

The author declares that he has no competing interests.

Published: 9 November 2010

References

1. Eyre S, Hinks A, Bowes J, Flynn E, Martin P, Wilson AG, Morgan AW, Emery P, Steer S, Hocking LJ, Reid DM, Harrison P, Wordsworth P, Yorkshire Early Arthritis (YEAR) Consortium, Biologics in RA Control (BIRAC) Consortium, Thomson W, Worthington J, Barton A: Overlapping genetic susceptibility variants between three autoimmune disorders: rheumatoid arthritis, type 1 diabetes and coeliac disease. Arthritis Res Ther 2010, 20:R175.

2. Wandstrat A, Wakeland $\mathrm{E}$ : The genetics of complex autoimmune diseases: non-MHC susceptibility genes. Nat Immuno/ 2001, 2:802-809.

3. Anaya JM, Corena R, Castiblanco J, Rojas-Villarraga A, Shoenfeld Y: The kaleidoscope of autoimmunity. Multiple autoimmune syndromes and familial autoimmunity. Expert Rev Clin Immunol 2007, 3:623-635.

4. Becker KG, Simon RM, Bailey-Wilson JE, Freidlin B, Biddison WE, McFarland HF, Trent JM: Clustering of non-major histocompatibility complex susceptibility candidate loci in human autoimmune diseases. Proc Natl Acad Sci U S A 1998, 95:9979-9984

5. Anaya JM, Gomez LM, Castiblanco J: Is there a common genetic basis for autoimmune diseases? Clin Dev Immunol 2006, 13:185-195.

6. Mamtani M, Anaya JM, He W, Ahuja SK: Association of copy number variation in the FCGR3B gene with risk of autoimmune diseases. Genes Immun 2010, 11:155-160.

7. Clarke A, Vyse TJ: Genetics of rheumatic disease. Arthritis Res Ther 2009, $11: 248$.

8. Delgado-Vega A, Sánchez E, Lofgren S, Castillejo-López C, Alarcón-Riquelme ME: Recent findings on genetics of systemic autoimmune diseases. Current 
Opinion Immunol 2010 Oct 6. [Epub ahead of print].

9. Liebman MN: Opening Pandora's box: clinical data and the study of complex diseases. Sci STKE 2002, 2002:pe20.

10. Visscher PM, Hill WG, Wray NR: Heritability in the genomics era-concepts and misconceptions. Nat Rev Genet 2008, 9:255-266.

11. Rojas-Villarraga A, Diaz FJ, Calvo-Páramo E, Salazar JC, Iglesias-Gamarra A, Mantilla RD, Anaya JM: Familial disease, the HLA-DRB1 shared epitope and anti-CCP antibodies influence time at appearance of substantial joint damage in rheumatoid arthritis. J Autoimmun 2009, 32:64-69.

12. Manolio TA, Collins FS, Cox NJ, Goldstein DB, Hindorff LA, Hunter DJ, McCarthy MI, Ramos EM, Cardon LR, Chakravarti A, Cho JH, Guttmacher AE,
Kong A, Kruglyak L, Mardis E, Rotimi CN, Slatkin M, Valle D, Whittemore AS, Boehnke M, Clark AG, Eichler EE, Gibson G, Haines JL, Mackay TF, McCarroll SA, Visscher PM: Finding the missing heritability of complex diseases. Nature 2009, 461:747-753.

doi:10.1186/ar3175

Cite this article as: Anaya J-M: The autoimmune tautology. Arthritis Research \& Therapy 2010, 12:147. 